Check for updates

Cite this: RSC Adv., 2017, 7, 49133

\title{
Colorimetric detection of epinephrine using an optimized paper-based aptasensor $\dagger$
}

\author{
Nileshi Saraf, ${ }^{a}$ Alexander Bosak, ${ }^{\mathrm{b}}$ Alicia Willenberg, ${ }^{\mathrm{b}}$ Soumen Das, ${ }^{\mathrm{a}}$ \\ Bradley Jay Willenberg ${ }^{\mathrm{b}}$ and Sudipta Seal (iD *a
}

A simple label-free colorimetric detection of epinephrine has been demonstrated based on its interaction with aptamer functionalized gold nanoparticles. The role of different parameters such as morphology of AuNPs, aptamer concentration and salt destabilization has been thoroughly investigated to enhance the performance of the sensor in terms of sensitivity and specificity. A range of common interfering agents such as 3,4-dihydroxyphenylacetic acid (DOPAC), tryptophan, dopamine, ascorbic acid etc. showed very little/no aggregation in the solution which indicates the specificity of the sensor. The sensor was then tested on a hydrophobic paper substrate for the development of a cheap, portable and point of care diagnostic tool. A visible change in colour from red to blue was observed in the solution with increasing concentration of epinephrine. The present approach gave a detection limit of $0.9 \mathrm{nM}$ using UV-visible spectroscopy which is by far the lowest detection limit of epinephrine using any colorimetric approach.

Received 15th September 2017

Accepted 14th October 2017

DOI: $10.1039 / \mathrm{c} 7 \mathrm{ra10272k}$

rsc.li/rsc-advances

environment and aggregation state. ${ }^{19-24}$ When AuNPs of $\sim 10-$ $20 \mathrm{~nm}$ size are uniformly dispersed in solution, they exhibit a strong plasmon peak at $\sim 520 \mathrm{~nm}$ due to the collective oscillations of the free electrons in the conduction band. Any change in physical parameters will cause a shift in the electron density, resulting in changes in oscillation frequency, absorption and scattering spectra and hence, the visible colour of the AuNPs solution from red to blue. This approach can be used to design simple biosensing devices to detect various kinds of molecules.

Aptamer-functionalized gold nanoparticles (AuNPs-apt) have also attracted attention for use in detecting small proteins, ${ }^{25-29}$ oligonucleotides ${ }^{19,30-32}$ and metal ions ${ }^{33-35}$ due to their low cost, simplicity and practicality. ${ }^{36-39}$ Aptamers are short, synthetic oligonucleotide strands of either DNA or RNA which are synthesized by a procedure called Systematic Evolution of Ligands by Exponential Enrichment (SELEX) and can be functionalized onto the surface of a nanoparticle. ${ }^{\mathbf{4 0 , 4 1}}$ These sensory molecules bind to target molecule by a lock and key mechanism driven by a conformational change as well as van der Waals forces, hydrogen bonding and electrostatic interactions. ${ }^{42}$ This binding cause energy/charge transfer between the AuNPs-apt and the target, leading to aggregation in the solution. The target-mediated aggregation of AuNPs-apt results in the colour change of the colloidal gold solution from red to blue. ${ }^{\mathbf{4 3 4} 4}$ This attribute of AuNPs of transferring any biorecognition event into a colour change has motivated scientists to use AuNPs as a biosensing tool for the detection of numerous biomolecules including oligonucleotides, small proteins, biomarkers and pathogens.

The catecholamine epinephrine (4-[(1R)-1-hydroxy-2(methylamino)ethyl]benzene-1,2-diol) is a neurotransmitter

${ }^{a}$ Advanced Materials Processing and Analysis Center, Department of Materials Science and Engineering, University of Central Florida, Orlando, FL-32816, USA. E-mail: Sudipta.Seal@ucf.edu; Tel: +1-4075928565

${ }^{b}$ Department of Internal Medicine, College of Medicine, University of Central Florida, Orlando, FL 32827, USA

$\dagger$ Electronic supplementary information (ESI) available. See DOI: $10.1039 / \mathrm{c} 7 \mathrm{ra} 10272 \mathrm{k}$ 
and neuroendocrine hormone which acts upon $\alpha$-adrenergic and $\beta$-adrenergic receptors in several organs, giving it a vast array of physiological functions. ${ }^{45}$ Clinical uses include the treatment of anaphylactic shock, cardiac attack and asthma attacks. ${ }^{45}$ Abnormalities in epinephrine levels may result in myocardial infarction, arrhythmias, pulmonary edema, blood pressure increase and Takotsubo cardiomyopathy. ${ }^{\mathbf{4 6 - 4 8}}$ The level of epinephrine present in the plasma is approximately $13.6 \times$ $10^{-11} \mathrm{M}^{49}$ Detection has previously been performed by electrochemical methods, ${ }^{50-52}$ liquid chromatography, ${ }^{53,54}$ spectrophotometry, ${ }^{55}$ electrophoresis ${ }^{56}$ flow injection analysis ${ }^{57,58}$ and fluorometry. ${ }^{59,60}$ The estimated detection limit for epinephrine obtained from all these experiments was found in the range of nanomolar to micromolar $\left(10^{-8}\right.$ to $\left.10^{-9} \mathrm{M}\right){ }^{49}$ Though these methods have high detection limits, they are time consuming and require skilled labour and expensive instrumentation. As an alternative, gold aptasensors not only provide a simple and novel testing tool but also demonstrates high sensitivity and selectivity towards epinephrine.

Despite having all the aforementioned advantages of using AuNPs-apt based colorimetric sensors, the biggest challenge which restricts the use of this sensor in real world application is the low sensitivity compared to other sensing technologies. The detection limit depends on the morphology of the AuNPs, the binding affinity of the aptamer, the ratio of the aptamer to the AuNPs and the affinity of the aptamer with respect to analyte. The shape, size and the dielectric medium surrounding the particles can be tuned such that even small amounts of analyte can trigger maximum aggregation/colour change. Along with nanospheres, various shapes such as nanorod and nanostars have been utilized for the development of colorimetric sensors. ${ }^{61-63}$ However, there have been no systematic studies done which compare the effects of different sizes and shapes of AuNPs and aptamer-AuNPs interaction on the detection limit of a sensor. Therefore, the present work explored the effect of different parameters that govern the detection process such as the number of aptamers on the surface of AuNPs, aptamer binding, salt concentration and physical morphology of AuNPs. The aim is to optimize all these parameters such that a maximum shift in the spectra and an intense change in colour can be obtained. These optimized parameters have been used to develop a sensitive and portable paper based sensor for epinephrine, which can be used as a point of care diagnostic tool.

\section{Experimental section}

\section{Materials}

Both thiol-modified and unmodified aptamers were used in the present study. The 32-mer aptamers specific for epinephrine were designed and synthesized by BasePairBio Company (Pearland, TX). Chloroauric acid, trisodium citrate, L-ascorbic acid, tris(2-carboxyethyl) phosphine (TCEP), tris hydrochloride (Tris $\mathrm{HCl}$ ), ethylenediaminetetraacetic acid (EDTA), phosphine buffered saline tablets, sodium chloride $(\mathrm{NaCl})$, magnesium chloride $\left(\mathrm{MgCl}_{2}\right)$ and epinephrine hydrochloride were purchased from Sigma-Aldrich (St. Louis, MZ). Gold nanospheres $(15 \mathrm{~nm}, 30 \mathrm{~nm}, 50 \mathrm{~nm})$ with concentration of $1 \mathrm{mg} \mathrm{mL} \mathrm{m}^{-1}$ and Gold NanoRods (660 nm resonant, $50 \mathrm{OD}$ ) stabilized by negatively charged citrate ions were obtained from Nanocomposix (San Diego, CA). Experiments were performed in a DNAse-RNAse free environment using nuclease free water, tips and vials purchased from Thermofisher (Waltham, MA). Whatman ${ }^{\circledR}$ filter paper \#1 was obtained from Sigma Aldrich (St. Louis, MZ).

\section{Instrumentation}

Surface charge and hydrodynamic size measurements were performed using Dynamic Light Scattering (DLS) by Zetasizer, Malvern Instrument equipped with $633 \mathrm{~nm} \mathrm{He-Ne} \mathrm{laser} \mathrm{and}$ a backscatter detector at an angle of $173^{\circ}$. Folded capillary tubes were used for measuring zeta potential at a controlled temperature of $25{ }^{\circ} \mathrm{C}$ and a fixed voltage of $150 \mathrm{~V}$. The $Z$-average size was calculated using the "General purpose" analysis model as provided by the Zetasizer software. For each sample, minimum of 3 measurements containing 11 runs of duration 10 seconds was performed. The results for the three readings were averaged and standard deviation was calculated to define the error bars on the DLS graphs. UV-vis spectroscopy was performed using FLUOstar Omega plate reader to determine the absorbance spectrum in the range of 400-800 $\mathrm{nm}$. UV transparent 96-well plates were used for the entire study. AuNPs based on their physical morphologies exhibit different absorbance peaks in the UV-spectrum. The size and morphology studies were also done using Philip (FEI Tecnai F30) high resolution transmission electron microscope (HRTEM) operating at $300 \mathrm{keV}$. The samples for TEM were prepared by dip-coating the carbon coated TEM grid in a diluted solution of the particles and drying it at room temperature. The steps were repeated multiple times to ensure enough AuNPs are present on the grid. The samples were stored under high vacuum overnight to remove any moisture and protect the sample from oxidation. The average size of the particles was calculated by using Gatan Digital Micrograph software.

\section{AuNPs synthesis}

AuNPs were synthesized using the standard citrate reduction procedure as reported in the literature previously. ${ }^{64}$ Briefly, $100 \mathrm{~mL}$ of $1 \mathrm{mM} \mathrm{HAuCl}{ }_{4}$ was heated to boil under constant stirring. Next, trisodium citrate solution ( $2 \mathrm{~mL}, 194 \mathrm{mM}$ ) was added rapidly in the stirring solution resulting in a change in solution colour from pale yellow to red. The solution was boiled for another $20 \mathrm{~min}$ and then allowed to cool at room temperature. The solution was then filtered using $0.22 \mu \mathrm{m}$ membrane filter to ensure the monodispersivity of the AuNPs and stored at $4{ }^{\circ} \mathrm{C}$ for long term stability. The concentration was calculated as $14.4 \mathrm{nM}$ with the help of Beer's law by using the extinction coefficient of $2.01 \times 10^{8} \mathrm{M}^{-1} \mathrm{~cm}^{-1} \cdot{ }^{65}$ The entire scheme is shown in Fig. 1.

\section{Preparation of AuNPs conjugated with aptamers}

The aptamers were received in a dried pelleted form and were resuspended in TE buffer $(10 \mathrm{mM}$ Tris $\mathrm{HCl}, 0.1 \mathrm{mM}$ EDTA, 


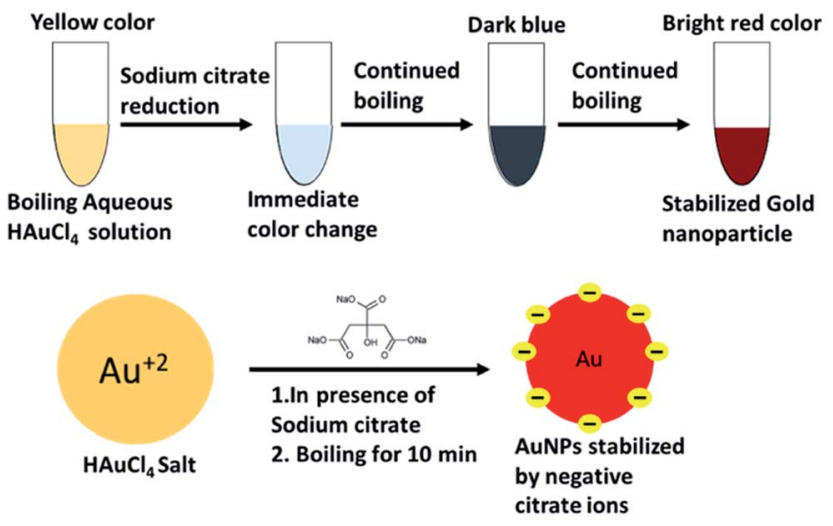

Fig. 1 Schematic of AuNPs synthesis using seedless approach.

$\mathrm{pH}$ 7.5) to achieve a concentration of $100 \mu \mathrm{M}$. The solution was aliquoted into smaller volumes to avoid multiple freeze and thaw cycles. Before immobilization of the aptamers over the AuNPs surface, the aptamers were diluted to the required concentration using folding buffer $\left(1 \mathrm{mM} \mathrm{MgCl}_{2}\right.$ and $0.01 \mathrm{M}$ PBS, pH 7.5). The aptamers were brought into a folded state by incubating at $95{ }^{\circ} \mathrm{C}$ for $5 \mathrm{~min}$ and another $15 \mathrm{~min}$ at room temperature. The thiolated aptamers were then reduced by using TCEP in $1: 1 \mathrm{v} / \mathrm{v}$ dilution. The addition of TECP ensures the breakage of any disulphide bonds (-S-S-) such that free $-\mathrm{SH}$ group is available to bind to the gold surface. The reaction was incubated for $20 \mathrm{~min}$ at room temperature. The unmodified aptamers did not require any reduction steps. AuNPs-aptamer complexes were prepared by mixing the AuNPs and folded aptamers in $1: 1 \mathrm{v} / \mathrm{v}$ ratio and kept at room temperature for another hour. Excess aptamers were washed away by centrifugation at $14000 \mathrm{rcf}$ for $11 \mathrm{~min}$ and resuspending the conjugates in folding buffer.

\section{Preparation of hydrophobic paper substrate}

Paper based substrate made up of cellulose, such as Whatman ${ }^{\circledR}$ filter paper are portable, easy to store, readily available and provide high surface area for colorimetric bioassays. The surface and pore size of the Whatman ${ }^{\circledR}$ filter paper can be easily functionalized and controlled by various chemical treatments as per requirement. ${ }^{66-68}$ In the present study, Whatman ${ }^{\circledR}$ filter paper was cut into strips and functionalized with wax by directly rubbing the solid wax over the paper. The paper was incubated for about an hour at $60{ }^{\circ} \mathrm{C}$ such that the wax spread evenly on the paper surface and seeped through the pores of the paper. The wax provides hydrophobic nature to the paper which does not allow AuNPs to wet the surface or spread on to the surface of paper. ${ }^{11}$

\section{Colorimetric assays}

$25 \mu \mathrm{L}$ of epinephrine at different concentrations was added to $50 \mu \mathrm{L}$ of AuNPs-apt solution for each colorimetric assay in the 96 well-plate. The amounts and concentrations of epinephrine used in the study were $2 \mu \mathrm{g}(360 \mu \mathrm{M}), 1 \mu \mathrm{g}(180 \mu \mathrm{M}), 500 \mathrm{ng}$ $(90 \mu \mathrm{M}), 350 \mathrm{ng}(63 \mu \mathrm{M}), 200 \mathrm{ng}(36 \mu \mathrm{M}), 125 \mathrm{ng}(22 \mu \mathrm{M}), 50 \mathrm{ng}$
$(9 \mu \mathrm{M})$ and so on. The AuNPs-apt and epinephrine solution was incubated for $10 \mathrm{~min}$ at room temperature before performing the UV-visible spectroscopy. For the different size and shape study, the concentrations of the different AuNPs were kept constant at $0.2 \mathrm{mg} \mathrm{mL}^{-1}$ for an accurate comparison of the detection limit. Each assay was performed at least in triplicate and the data is represented as mean \pm standard errors.

\section{Results and discussions}

\section{AuNPs characterization}

Fig. 2 shows detailed characterization of the as-synthesized (13 $\mathrm{nm}$ spherical particles) and purchased AuNPs. AuNPs $(13 \mathrm{~nm})$ were synthesized using the standard procedure of citrate reduction of $\mathrm{HAuCl}_{4}$ as shown in Fig. 1. The hydrodynamic radii of the gold colloids are $18.15 \mathrm{~nm}, 20.10 \mathrm{~nm}$, $36.48 \mathrm{~nm}, 52.47 \mathrm{~nm}$ and $55.91 \mathrm{~nm}$ as measured with DLS instrument and it can be seen that all the particles are in a very narrow range with minimum/no agglomeration (Fig. 2A). Zeta potential results (Fig. 2B) indicate that the citrate coated particles are negatively charged with surface charge as $-21.9 \mathrm{mV}$, $-28.33 \mathrm{mV},-37.8 \mathrm{mV},-52.2 \mathrm{mV}-52.5 \mathrm{mV}$ and $-43.9 \mathrm{mV}$ and this charge increases with respect to the size of the particles. The negative charge of AuNPs induces stability in the solution and prevents unwanted agglomeration. AuNPs of diameter $13 \mathrm{~nm}, 15 \mathrm{~nm}, 30 \mathrm{~nm}$ and $50 \mathrm{~nm}$ stabilized in water exhibited distinctive peaks (surface plasmon band, SPR) at around
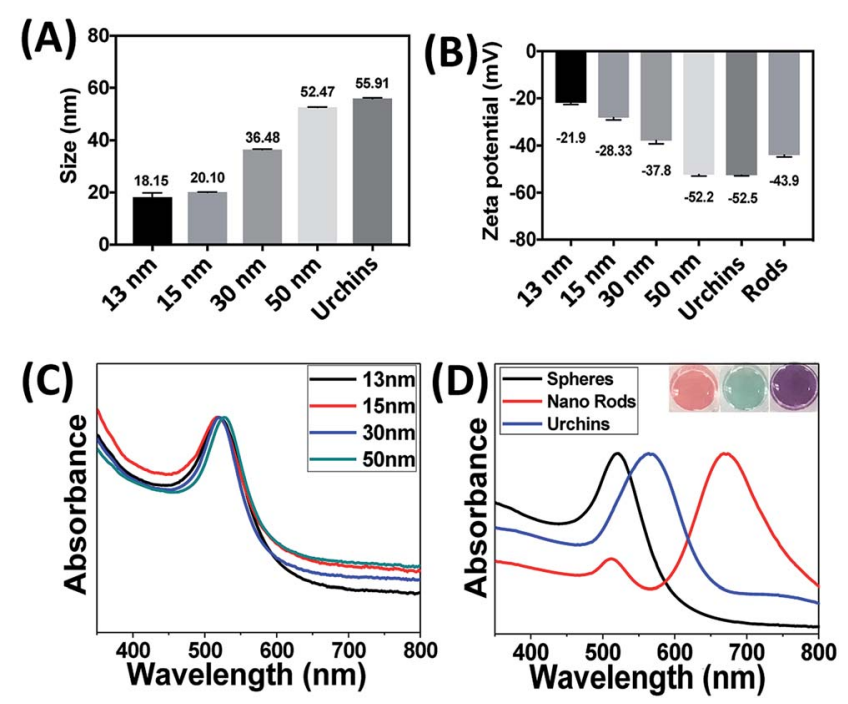

Fig. 2 Thorough characterization of different kind of AuNPs. (A) Dynamic light scattering measurements showing the diameter of the spherical particles as well as nanourchins. The mean sizes observed are $18.15 \mathrm{~nm}, 20.10 \mathrm{~nm}, 36.48 \mathrm{~nm}, 52.47 \mathrm{~nm}$ and $55.91 \mathrm{~nm}$ respectively. (B) Surface zeta potential of the given samples shows the presence of negative charge with a small error bar. (C) Normalized UV-visible spectra of the spherical particles with SPR bands at $518 \mathrm{~nm}, 520 \mathrm{~nm}$, $524 \mathrm{~nm}$ and $528 \mathrm{~nm}$. (D) UV-visible spectra of different shapes of AuNPs. The maxima was observed at $518 \mathrm{~nm}, 562 \mathrm{~nm}$ for spheres and urchins whereas for rods, these were $520 \mathrm{~nm}$ and $670 \mathrm{~nm}$. The inset shows the visible colour of different shapes - spheres (red), rods (green) and nanourchins (purple). 
$518 \mathrm{~nm}, 520 \mathrm{~nm}, 524 \mathrm{~nm}$ and $528 \mathrm{~nm}$ respectively (Fig. 2C). The SPR of the AuNPs is modified when the shape deviates from spherical geometry. ${ }^{69}$ In the case of rods, the electrons are found to oscillate in the transverse as well as longitudinal direction. The transverse mode showed a resonance peak at approximately $520 \mathrm{~nm}$ whereas the longitudinal direction peak was observed at $670 \mathrm{~nm}$ (Fig. 2D). Nanourchin shaped particles exhibited a broad, shifted peak at $\sim 562 \mathrm{~nm}$ due to the presence of spikes which results in complex localized surface plasmon resonance (LSPR). Different absorption peaks of these particles cause changes in their visible colour (Fig. 2D).

High resolution transmission electron microscopy (HRTEM) images of the as prepared and purchased nanoparticles are shown in Fig. 3(A-F). HRTEM images revealed that particles are uniformly dispersed and have spherical, spiky and rod-like morphologies. The average sizes are in order of $12.74 \mathrm{~nm}$, $14.66 \mathrm{~nm}, 27.77 \mathrm{~nm}, 48.71 \mathrm{~nm}, 53.76 \mathrm{~nm}$ and $45.34 \mathrm{~nm}$ (Table 1). The next section discusses the effect of aptamer concentration as well as the binding mechanism on the aggregation of the AuNPs.

\section{Effect of aptamer concentration}

$13 \mathrm{~nm}$ AuNPs were conjugated with different concentrations of the epinephrine-binding aptamer $(2 \mu \mathrm{M}, 200 \mathrm{nM}, 20 \mathrm{nM}, 2 \mathrm{nM}$, $0.2 \mathrm{nM}$ and $0.02 \mathrm{nM}$ ) and tested against a range of concentrations of epinephrine $(2 \mu \mathrm{g}(360 \mu \mathrm{M}), 1 \mu \mathrm{g}(180 \mu \mathrm{M}), 500 \mathrm{ng}$ $(90 \mu \mathrm{M}), 350 \mathrm{ng}(63 \mu \mathrm{M}), 200 \mathrm{ng}(36 \mu \mathrm{M})$ and $50 \mathrm{ng}(9 \mu \mathrm{M}))$. Fig. 4 shows the visual colour change at different aptamer concentration in the presence of epinephrine and the corresponding UV-visible spectra of these samples is represented in Fig. S1 (see ESI $\dagger$ ). The destabilization of the charge causes aggregation in the solution which can be seen from the TEM analysis as shown in Fig. S2 (see ESI†).

The change in colour was observed in solutions starting from $200 \mathrm{ng}$ of epinephrine and aptamer concentration ranging from $0.02 \mathrm{nM}$ to $20 \mathrm{nM}$. It was observed that the SPR of AuNPs at $520 \mathrm{~nm}$ broadens and shifts to a longer wavelength as a function of the epinephrine concentration. The degree of aggregation is represented in terms of $A_{640} / A_{518}$ where $A_{640}$ and $A_{518}$ indicate the absorbance intensity at $640 \mathrm{~nm}$ and $518 \mathrm{~nm}$, respectively. Fig. 5 demonstrates a comparative analysis of different samples. It can be seen that the increase in aptamer concentration ( $20 \mathrm{nM}, 200 \mathrm{nM}$ and $2 \mu \mathrm{M}$ ) decreases the detection limit of the AuNPs-apt system. There was no aggregation observed in the $2 \mu \mathrm{M}$ aptamer solution at any epinephrine concentration.

This result of decreased detection with increased aptamer concentration is due to the fact that as epinephrine is introduced into the solution, the free unbound aptamer binds to it. ${ }^{70}$ Since the epinephrine molecule is very small in size (Molecular Weight: $219.67 \mathrm{Da}$ ), the aptamer wraps around the molecule, making it very difficult for the molecule to interact with the other aptamers present on the gold surface, thus inhibiting aggregation of the AuNPs. Also, the conjugated aptamers add to
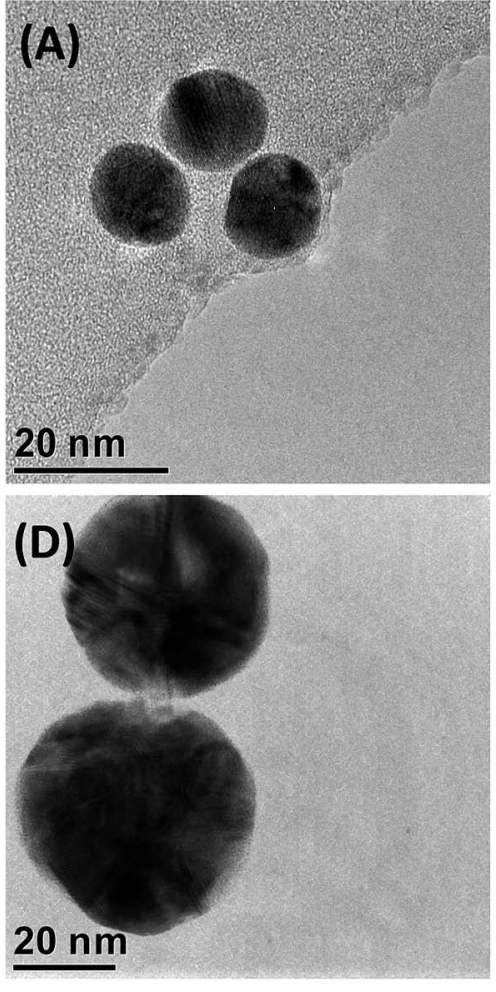
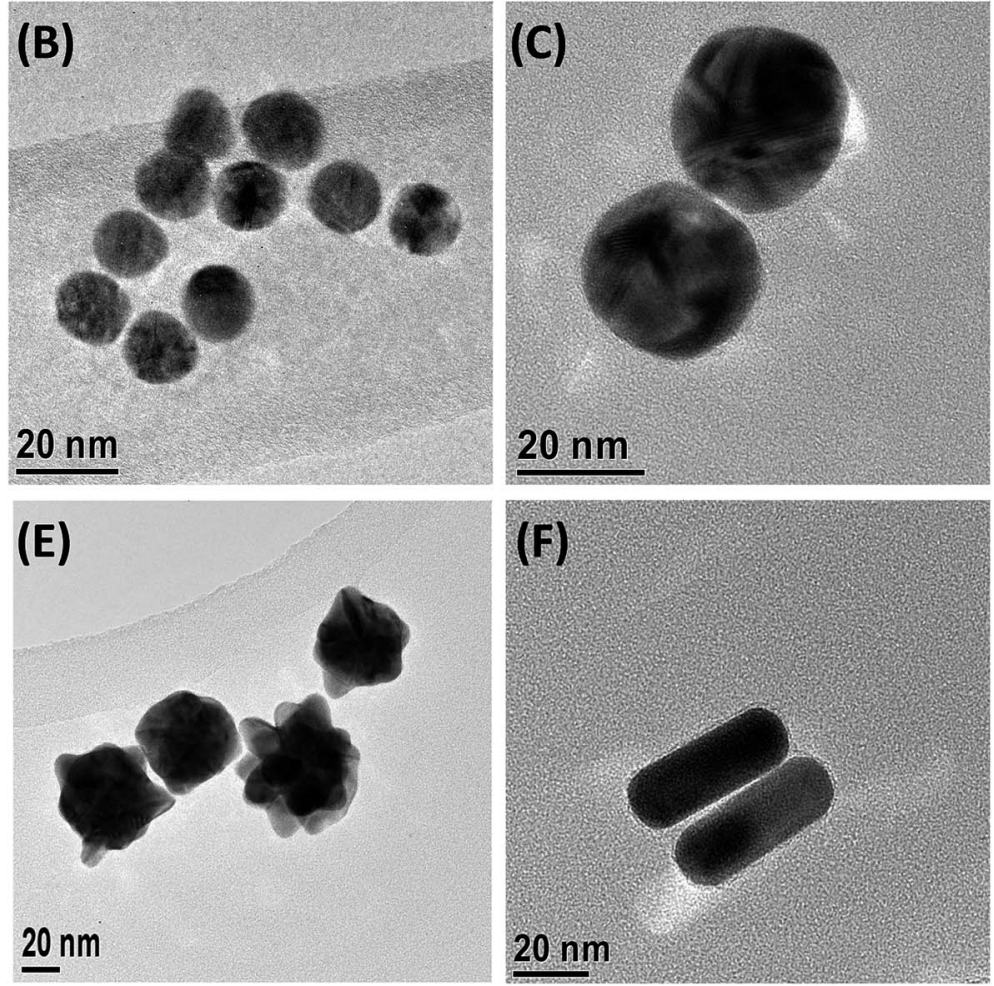

Fig. 3 High Resolution Transmission Electron Microscope analysis for different sizes and shapes of gold nanoparticles. (A-F) Images show the TEM for $13 \mathrm{~nm}, 15 \mathrm{~nm}, 30 \mathrm{~nm}, 50 \mathrm{~nm}$, nanourchins and nano rods respectively. The length is calculated using Digital Micrograph software provided by Gatan. 
Table 1 Summary of the properties of gold nanoparticles as characterized through various techniques

\begin{tabular}{lllll}
\hline Gold nanoparticles & Zeta potential $(\mathrm{mV})$ & SPR band $(\mathrm{nm})$ & $\begin{array}{l}\text { Hydrodynamic size } \\
(\mathrm{nm})\end{array}$ & TEM average size $(\mathrm{nm})$ \\
\hline Sphere $-13 \mathrm{~nm}$ & -21.9 & 518 & $18.15 \pm 2.1$ & $12.74 \pm 0.5$ \\
Sphere $-15 \mathrm{~nm}$ & -28.33 & 520 & $20.10 \pm 0.6$ & $14.66 \pm 0.3$ \\
Sphere $-30 \mathrm{~nm}$ & -37.8 & 524 & $36.48 \pm 0.3$ & $27.77 \pm 1.2$ \\
Sphere $-50 \mathrm{~nm}$ & -52.2 & 528 & $52.47 \pm 0.4$ & $48.71 \pm 2.5$ \\
Nanourchin & -52.5 & 562 & $55.91 \pm 0.4$ & $53.76 \pm 6.3$ \\
Nano rods & -43.9 & 520,620 & - & $45.34 \pm 0.4$
\end{tabular}

the overall negative charge of the AuNPs, enhancing the stability of the AuNPs by increasing the electrostatic repulsion between the particles. ${ }^{71,72}$ It can be observed that the optimized range of aptamer for maximum detection of the analyte is below $20 \mathrm{nM}$ aptamer concentration which can detect epinephrine levels as low as $200 \mathrm{ng}$. At lower concentration of aptamers, a linear increase in the ratio was observed at $50 \mathrm{ng}, 200 \mathrm{ng}$ and $350 \mathrm{ng}$ of epinephrine which becomes constant at higher concentrations of epinephrine. $2 \mathrm{nM}$ aptamer concentration gave the maximum $A_{640} / A_{518}$ ratio which suggest that it is the optimum aptamer concentration at which maximum aggregation was seen in the solution at $200 \mathrm{ng}$ of epinephrine. Higher concentration of aptamer induces excess stability in the solution as discussed previously. The aptamer concentration lower than $2 \mathrm{nM}$ i.e. $0.2 \mathrm{nM}$ and $0.02 \mathrm{nM}$ is insufficiently low in number and in the presence of low concentration of the epinephrine molecule, not enough aptamers are present to induce aggregation in the AuNPs solution. Therefore, AuNPs are conjugated with $2 \mathrm{nM}$ aptamer concentration for further experiments. The aptamers can be physically adsorbed ${ }^{72}$ on the surface of AuNPs or can bind to the surface of AuNPs via covalent bonding. ${ }^{73,74}$ The following experiment compares the two different types of the aptamers and studies their effect on the aggregation.

\section{Effect of thiolated and non-thiolated aptamer conjugation}

Aptamers can bind to the surface via covalent bond (Au-thiol interactions $)^{73,74}$ or it can be adsorbed on the surface via

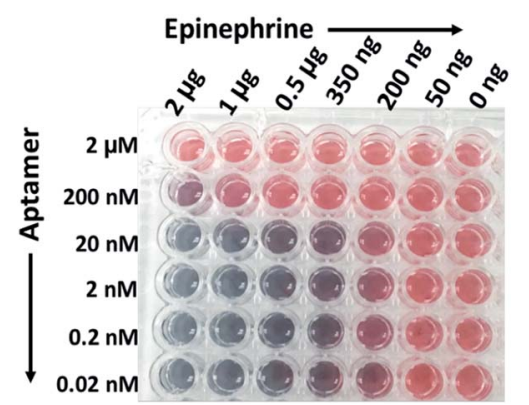

Fig. 4 Visible colour change in the solution of AuNPs conjugated with different concentration of thiolated aptamers $(0.02 \mathrm{nM}$ to $2 \mu \mathrm{M})$ in presence of epinephrine. The change in colour represents aggregation in the solution. The AuNPs started aggregating when the aptamer concentration was $20 \mathrm{nM}$ or less and minimum concentration of epinephrine was $200 \mathrm{ng}$. electrostatic interactions. ${ }^{72}$ Fig. 6 represents the comparison between the thiolated and non-thiolated epinephrine aptamer and its effect on the sensitivity of the aptasensor. As it was observed earlier that $200 \mathrm{ng}$ of epinephrine induced intense colour change in the solution and red-shift in the UV spectrum, the AuNPs-aptamers were now tested against $125 \mathrm{ng}$ of epinephrine. It can be seen from Fig. S2(A-C), $\dagger 125 \mathrm{ng}$ induced slight colour change in the solutions in the presence of aptamer ranging from $20 \mathrm{nM}$ to $0.02 \mathrm{nM}$. However, the UV-visible data (Fig. S3, ESI $\dagger$ ) shows the highest peak at $640 \mathrm{~nm}$ which belonged to $2 \mathrm{nM}$ aptamer. The maximum $A_{640} / A_{518}$ ratio was achieved for $2 \mathrm{nM}$ of the thiolated aptamer as shown in Fig. 6. The thiolated and non-thiolated produced similar results in terms of colour change but UV-visible data showed that the thiolated aptamers are more efficient in detecting the analyte.

The variation in detection of analyte by thiolated versus nonthiolated aptamers is due to differences in the underlying mechanisms of these two approaches. Thiolated aptamers bind strongly to the surface of the gold and when the analyte approaches, AuNPs are pulled together strongly to form aggregates. ${ }^{75}$ The repulsion between the citrate capped AuNPs is dominated by the affinity of aptamers towards the analyte. The presence of epinephrine bound with the aptamers causes destabilization in the charge of AuNPs which results in

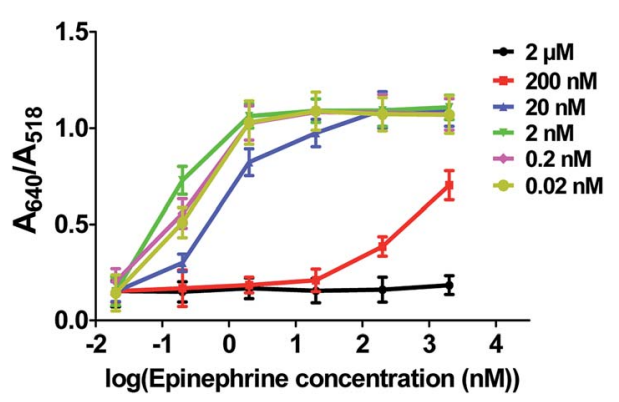

Fig. 5 Comparative analysis of the $A_{640} / A_{518}$ ratio as derived from UV-visible spectroscopy analysis of samples containing different amount of aptamers ( $2 \mu \mathrm{M}, 200 \mathrm{nM}, 20 \mathrm{nM}, 2 \mathrm{nM}, 0.2 \mathrm{nM}$ and $0.02 \mathrm{nM})$ as a function of epinephrine concentration represented in logarithmic scale $(2 \mu \mathrm{g}(360 \mu \mathrm{M}), 1 \mu \mathrm{g}(180 \mu \mathrm{M}), 500 \mathrm{ng}(90 \mu \mathrm{M}), 350 \mathrm{ng}(63 \mu \mathrm{M})$, $200 \mathrm{ng}(36 \mu \mathrm{M})$ and $50 \mathrm{ng}(9 \mu \mathrm{M}))$. The increase in the ratio is in correspondence with the colour change in the solution. Solution with $2 \mathrm{nM}$ concentration (green curve) shows the most significant increase in the ratio at $200 \mathrm{ng}$ of epinephrine which indicates maximum aggregation in the solution. 


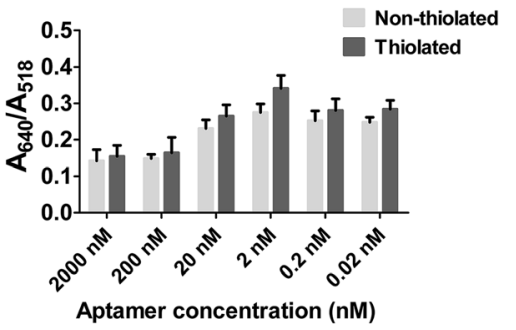

Fig. 6 Comparative analysis of thiolated and non-thiolated aptamer on the surface of AuNPs in the presence of $125 \mathrm{ng}$ of epinephrine. The absorbance ratio $\left(A_{640} / A_{518}\right)$ as calculated by UV-visible spectra represents maximum aggregation in $2 \mathrm{nM}$ thiolated aptamer solution. The columns represent the mean value and the error bars indicate the standard deviation in the experiments.

aggregation/colour change. On the other hand, the unmodified aptamers are loosely attached to the AuNPs surface. In the presence of epinephrine, the aptamers attain a tertiary folded conformation and detach themselves from the surface, leaving the AuNPs destabilized. ${ }^{76,77}$ The extent of destabilization depends on the concentration of analyte and the number of aptamers detached from the surface. The lesser shift in the peak at $\sim 640 \mathrm{~nm}$ in the presence of unmodified aptamers is due to aptamers remaining attached to the surface of AuNPs and screening them from aggregation. However, the difference is very minor, and therefore, it can be concluded that both thiolated and non-thiolated aptamers behave almost the same for low epinephrine concentrations. Moving forward, thiolated aptamers were used at $2 \mathrm{nM}$ concentration for testing the effect of AuNPs physical morphology on the detection of epinephrine.

\section{Effect of different sizes}

So far, the effect of aptamer concentration and the thiolated and non-thiolated binding of the aptamers has been discussed. $2 \mathrm{nM}$ thiolated aptamer showed maximum sensitivity and visible colour change towards $125 \mathrm{ng}$ of epinephrine with a maximum peak intensity at $640 \mathrm{~nm}$. Different sizes of spherical gold nanoparticles ( $13 \mathrm{~nm}, 15 \mathrm{~nm}, 30 \mathrm{~nm}$ and $50 \mathrm{~nm}$ ) have been used to test the limit of detection of the aptasensor. Fig. 7(A and B) shows the response of different sizes of AuNPs in the presence of $125 \mathrm{ng}$ and $200 \mathrm{ng}$ of epinephrine. The
UV-visible spectrum shows that the $13 \mathrm{~nm}$ AuNPs (black curve) exhibited a greater red shift in the presence of $125 \mathrm{ng}$ and $200 \mathrm{ng}$ epinephrine compared to other particles. No shift was seen for $30 \mathrm{~nm}$ and $50 \mathrm{~nm}$ AuNPs. The derived absorbance ratio $\left(A_{640} / A_{518}\right)$ from the UV-visible spectra (Fig. 7C) confirms that the maximum aggregation was observed for $13 \mathrm{~nm}$ particles, followed by $15 \mathrm{~nm}$ particles with little to no shift for $30 \mathrm{~nm}$ and $50 \mathrm{~nm}$ AuNPs. $13 \mathrm{~nm}$ AuNPs also exhibited the maximum colour change for all tested concentration of epinephrine (Fig. S4†).

The strongly bound citrate group induces negative charge on the surface of the AuNPs which leads to repulsion between the particles. ${ }^{78}$ Since the $50 \mathrm{~nm}$ AuNPs have the maximum negative surface charge (Table 1), very little aggregation was observed in the solution. Because larger sized particles are bulkier than smaller ones, higher concentrations of analyte are required for the aptamers to agglomerate the particles. The combined effect of the negative zeta potential as well as the interaction of aptamer and analyte is responsible for no colour change in larger particles.

\section{Effect of different shapes: rods, spheres and urchins}

Anisotropic nanomaterials with highly complex structures have generated wide spread interest owing to their enhanced physical and optical properties. In the present section, a comparative analysis of different shapes of AuNPs used for the detection of epinephrine has been carried out and the basic characterization is shown in Fig. 2 and 3. The aptamer conjugation process and the concentration of all AuNPs was kept constant as used for the spherical particles. Fig. 8(A-D) summarizes the result of the assay. The UV-visible spectra showed an intense red shift in the peak of spherical particles (Fig. 8A). The maximum shift was seen by addition of $500 \mathrm{ng}$ of epinephrine followed by $250 \mathrm{ng}$ and $125 \mathrm{ng}$. The colour change is also in line with the UV shift with maximum change caused by adding $500 \mathrm{ng}$ of epinephrine. In the case of rods, there was no shift in the peaks or change in colour. The intensity at peak $670 \mathrm{~nm}$ showed a constant decrease with respect to the epinephrine concentration and a very slight shift towards a lower wavelength. In rods, the red or blue shift is a result of change in the aspect ratio. The slight blue shift is due to the decrease in the aspect ratio which indicates that some of the rods have aggregated due to epinephrine. However, there was no change in colour observed
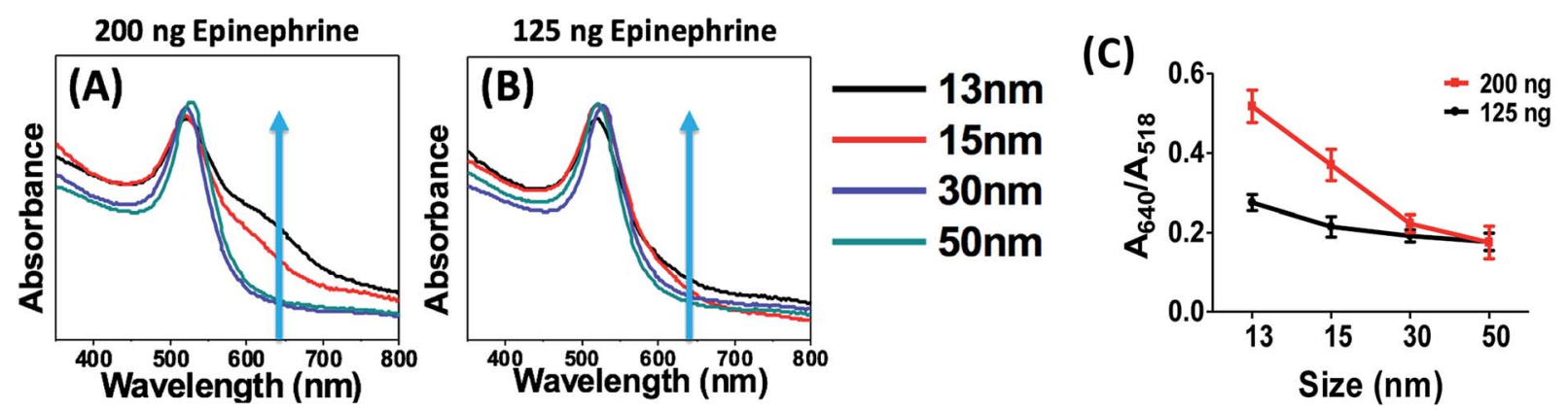

Fig. 7 Size dependent study for the detection of epinephrine. (A and B) UV-visible spectra showing aggregation in the solution of different sizes $\left(13 \mathrm{~nm}, 15 \mathrm{~nm}, 30 \mathrm{~nm}\right.$ and $50 \mathrm{~nm}$ ) of AuNPs at $125 \mathrm{ng}$ and $200 \mathrm{ng}$ of epinephrine. (C) Plot showing trend for the derived absorbance ratio ( $A_{640} /$ $\left.A_{518}\right)$ for different sized particles. $13 \mathrm{~nm}$ particles showed maximum aggregation in presence of $200 \mathrm{ng}$ and $125 \mathrm{ng}$ of epinephrine. 

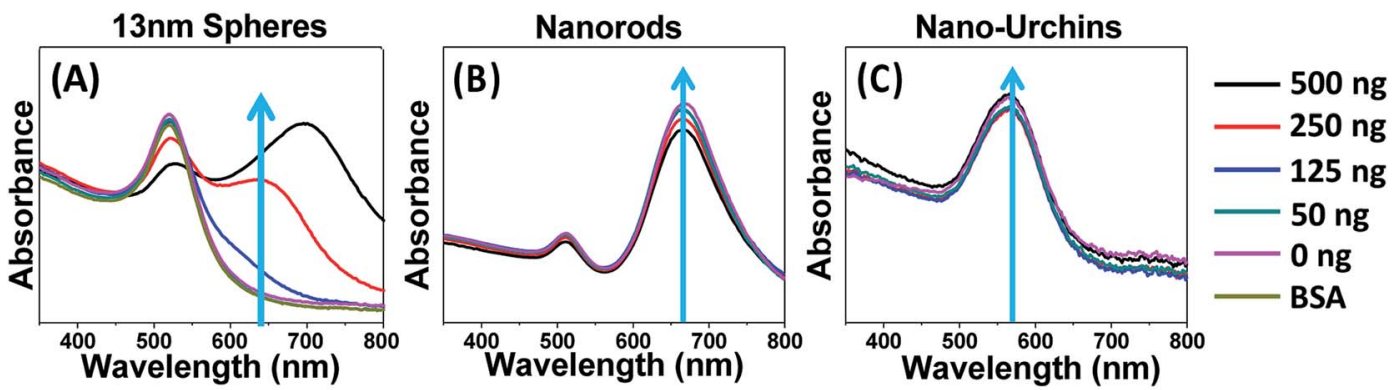

(D)

\section{Epinephrine}

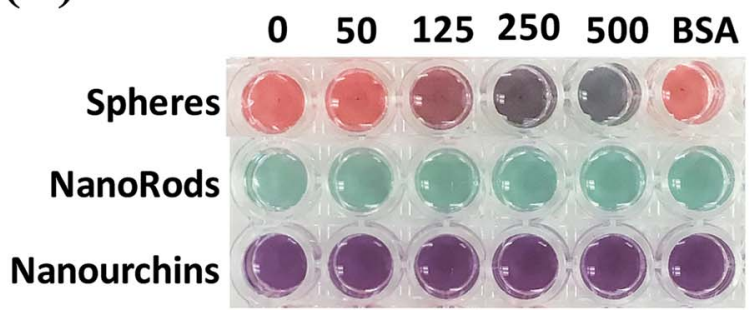

Fig. 8 Detection of epinephrine using different shapes of AuNPs. A, B and C represents the change in UV visible spectra in presence of 13 nm spherical AuNPs, gold nanorods and $50 \mathrm{~nm}$ nano urchin. Spherical particles show clear red shift at 500, 250 and $125 \mathrm{ng}$ of epinephrine whereas in case rods and nanourchin, very slight change in the intensity of the peaks can be seen. The colour change as shown in $D$ is prominent for spherical particles whereas no colour change was observed for rods and urchin.

for the rods. Nanourchins on the other hand showed no change in the UV-visible spectra or visible colour following the addition of epinephrine. As observed earlier, bigger size and greater negative charge leads to stability of the particles and lowers the detection limit. The rods and urchins both have sizes in the range of $50 \mathrm{~nm}$ and the zeta potential is also higher as compared to $13 \mathrm{~nm}$ spherical particles (Table 1). Also, the aggregation behaviour of the rods and spheres are different from each other. Nanorods are more stable than the spherical particles as nanorods are known to possess steric hindrance which causes low packing density and smaller aggregates. ${ }^{7-81}$ The spheres and urchins both have higher curvature, thus higher packing density; however, the spiky structure disrupts aggregation of urchins. Therefore, spherical particles with size between $13-15 \mathrm{~nm}$ are the most suitable particles for the colorimetric sensor.

\section{Selectivity of the sensor}

The specificity of the present colorimetric sensor was tested by using common interfering agents such as 3,4-dihydroxyphenylacetic acid (DOPAC), ascorbic acid, tryptophan, homovanillic acid (HVA), tyrosine, glycine and bovine serum albumin (BSA). $900 \mu \mathrm{M}$ of control samples were tested against $90 \mu \mathrm{M}$ (500 ng) epinephrine to test the specificity of AuNPs-apt. Maximum shift in UV-spectroscopy is obtained by the addition of epinephrine, with very little shift being induced by dopamine and no shift by other controls (Fig. 9). These results demonstrate the high specificity of this colorimetric biosensor against epinephrine.

\section{Aggregation study-time based study}

The aggregation kinetics was studied by examining the rate of aggregation with respect to the analyte concentration. Two different concentrations of epinephrine (200 $\mathrm{ng}$ and $125 \mathrm{ng}$ ) were used to induce aggregation in the solution and the size of the aggregates was calculated using DLS. $200 \mathrm{ng}$ of epinephrine induced immediate aggregation in the solution. The change in size in the presence of $200 \mathrm{ng}$ and $125 \mathrm{ng}$ of epinephrine at different time intervals $(0 \mathrm{~min}, 10 \mathrm{~min}, 30 \mathrm{~min}, 1$ hour, 2 hours and 3 hours) is shown in Fig. 10. It can be seen that in presence of $200 \mathrm{ng}$ of epinephrine, the solution started showing an increase in size immediately with the initial size of $21.38 \mathrm{~nm}$ as compared to the $18.16 \mathrm{~nm}$ of $125 \mathrm{ng}$ epinephrine. A gradual increase in size can be observed for $200 \mathrm{ng}$ of epinephrine; however, no change in size of AuNPs-apt was observed in the presence of $125 \mathrm{ng}$ for almost half an hour (Fig. 10). After 1 hour, the size increased from $18.16 \mathrm{~nm}$ to $31.21 \mathrm{~nm}$. For both samples, the size remained constant after 2 hours. This is in compliance with the established studies in the literature which

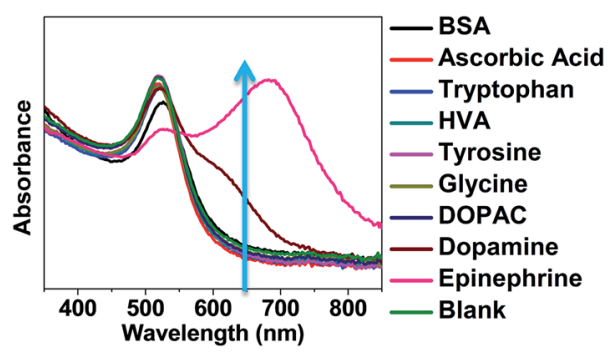

Fig. 9 Specificity test: UV-visible spectra of AuNPs conjugated with $2 \mathrm{nM}$ aptamer at $900 \mu \mathrm{M}$ concentration of interfering agents such as BSA, ascorbic acid, tryptophan, HVA, tyrosine, glycine, DOPAC and dopamine. Epinephrine (500 ng) caused maximum shift in the UV-visible spectra. Control represents the sample without any analyte in the solution. 


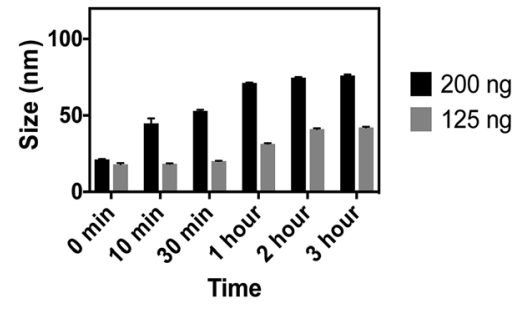

Fig. 10 Comparative analysis of the change in size of AuNPs in the presence of $200 \mathrm{ng}$ (black) and $125 \mathrm{ng}$ (gray) of epinephrine.

suggest that this is due to the completion of charge transfer and stabilization of the aggregates. ${ }^{78}$ When the interparticle distance is greater than the average radius of the particles, the particles are uniformly dispersed in the solution and emits red colour whereas when the interparticle distance is less than the average radius, the solution starts changing to blue colour. After 24 hours, the aggregated particles precipitated in the plate and showed no further increase in size.

\section{Effect of salt}

As discussed previously, the surface of the synthesized AuNPs is derivatized with citrate ligands which provide them stability against aggregation. Increases in salt concentration increase the ionic strength of a solution which results in screening of this repulsion force and induction of aggregation/flocculation of AuNPs. When the AuNPs are conjugated with aptamers, the aptamers protect the surface of the AuNPs against salt induced aggregation. However, the presence of epinephrine bound to the aptamer decreases the overall negative charge of the AuNPs and causes destabilization of the apt-AuNPs. Addition of salt further amplifies the charge destabilization and causes aggregation in solution.

The present AuNPs-aptamer system was tested against a range of $\mathrm{NaCl}$ concentration and it was observed that $170 \mathrm{mM}$ $\mathrm{NaCl}$ induced no shift in the UV spectra and was therefore chosen for further experiments. Fig. 11A shows the UV-visible spectra of the AuNPs-aptamer in the presence of different concentration of epinephrine after the addition of $170 \mathrm{mM}$

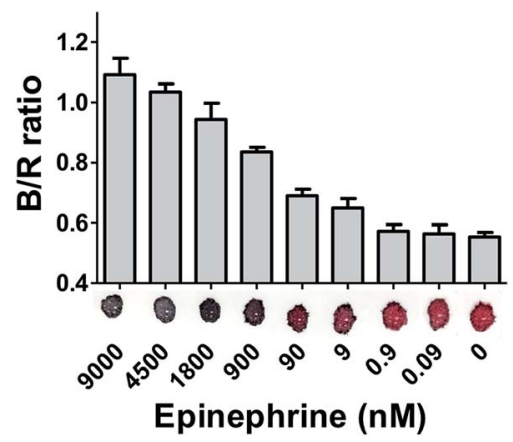

Fig. 12 Colorimetric analysis of AuNPs-aptamer in the presence of different concentration of epinephrine $(9 \mu \mathrm{M}, 4.5 \mu \mathrm{M}, 1.8 \mu \mathrm{M}, 9 \mu \mathrm{M}$, 900 nM, 90 nM, 9 nM, 900 pM, 90 pM) on a hydrophobic paper based substrate. The corresponding ratio of blue/red colour values of the AuNPs spots are represented in the figure. The columns represent the mean values whereas the small error bars represent the standard deviation in the different set of experiments. The change in colour was observed at $9 \mathrm{nM}$ with a significant increase in $B / R$ value from 0.58 to 0.65

$\mathrm{NaCl}$. There is a clear red shift in the spectra with the increase in the concentration of epinephrine. The derived absorbance ratio $\left(A_{640} / A_{518}\right)$ increases proportionally from 0.22 to 1.15 with increase in the epinephrine concentration which corresponds to aggregation in the solution (Fig. 11B). The lowest amount of epinephrine that induced red shift in the spectra was $0.005 \mathrm{ng}$ (0.9 nM) as shown in Fig. 11. In the absence of epinephrine, the aptamers conjugated AuNPs show high stability against $\mathrm{NaCl}$ and no red shift was observed in the UV-Visible spectra. The present approach with the optimized parameters was then tested on a hydrophobic paper substrate to formulate an easyto-use device.

\section{Paper based sensor}

So far, it has been discussed that $13 \mathrm{~nm}$ spherical particles when conjugated with $2 \mathrm{nM}$ thiolated aptamers in the presence of salt showed high detection limit for epinephrine (0.9 nM). The increase in concentration of epinephrine leads to increase
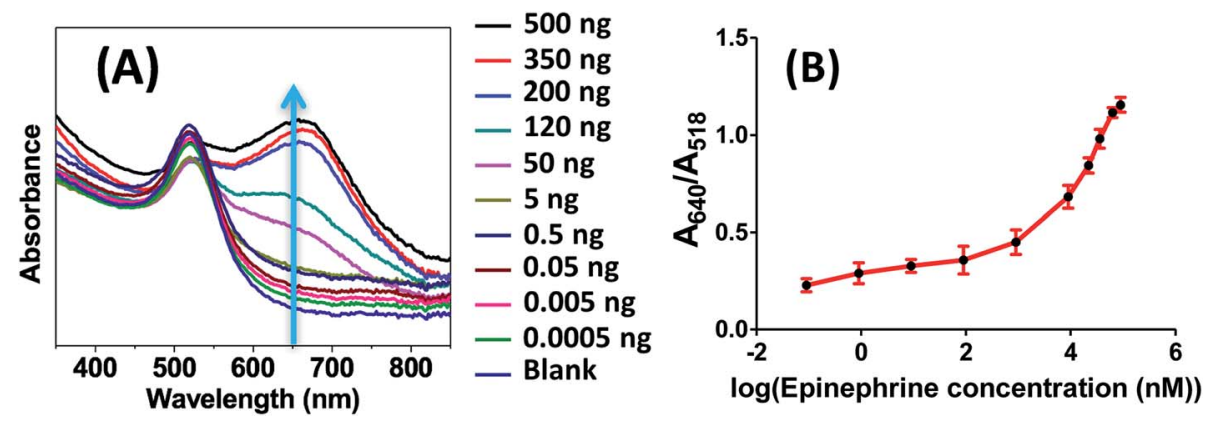

Fig. 11 Effect of addition of salt on the detection limit of the sensor. (A) UV-visible spectroscopy showing spectral shift in the presence of difference concentration of epinephrine ranging from $0.5 \mathrm{pg}$ to $500 \mathrm{ng}(0.09 \mathrm{nM}$ to $90 \mu \mathrm{M})$ and salt (170 mM). Addition of salt causes red shift in the spectra in the presence of epinephrine whereas no shift was seen for the blank sample (no analyte). (B) The derived absorbance ratio $\left(A_{640} /\right.$ $\left.A_{518}\right)$ increases proportionally with the increase in epinephrine concentration. Lowest concentration of epinephrine that induced red shift in the UV-visible spectra is $0.9 \mathrm{nM}$. 
in aggregation and thus increases the intensity of colour change as seen from the shift in UV-visible spectra. The AuNPs-aptamer based biosensor with all the optimized parameters were then used for the fabrication of a paper based sensor.

The colour change on the paper was calculated quantitatively using the red-green-blue values of each AuNPs spot determined by image processing software (Color helper, 2013). ${ }^{82}$ The blue/ red ratio directly correlates with the degree of aggregation in the solution and hence the concentration of epinephrine. $10 \mu \mathrm{L}$ of AuNPs-aptamer was added on the surface of the paper and different concentrations of epinephrine $(9 \mu \mathrm{M}, 4.5 \mu \mathrm{M}, 1.8 \mu \mathrm{M}$, $9 \mu \mathrm{M}, 900 \mathrm{nM}, 90 \mathrm{nM}, 9 \mathrm{nM}, 900 \mathrm{fM}, 90 \mathrm{fM}$ ) were added onto the drop of AuNPs solution. To enhance the aggregation in the solution, $2 \mu \mathrm{L}$ of $170 \mathrm{mM} \mathrm{NaCl}$ was also added in all the samples which resulted in immediate colour change (Fig. 12). The addition of as low as $9 \mathrm{nM}$ epinephrine began inducing colour change in the solution and resulted in a significant increase in the $\mathrm{B} / \mathrm{R}$ ratio. The $\mathrm{B} / \mathrm{R}$ value for AuNPs without aggregation in the solution was found to be around 0.58 and addition of epinephrine increases this value to more than 1 which indicates aggregation of AuNPs-apt in solution.

The developed paper based sensor can be used in conditions where plasma catecholamines reach supraphysiological levels. In patients with stress induced cardiomyopathy (Takotsubo cardiomyopathy) and Killip Class III myocardial infarction, epinephrine levels average $6.9 \times 10^{-9} \mathrm{M}$ and $2.1 \times 10^{-9} \mathrm{M}$ after 1 or 2 days, respectively. $^{83}$ Normal physiological serum epinephrine concentration is approximately $0.13 \times 10^{-9} \mathrm{M}$, which is below the detection limit of the sensor. ${ }^{49}$ Therefore, supraphysiological levels of epinephrine will induce a colour shift on the optimized paper sensor detectable with UV-visible spectroscopy but normal physiological values will not. This supports the diagnostic use of the sensor in a real-world situation. Furthermore, since the UV-vis shift is correlated to the concentration of target analyte, this method may also be used as a quick determinant of epinephrine levels in the patient.

The use of a cellulose-based paper approach provides a simple, easy and equipment free tool for sensing biomolecules. Of note, the paper did not interfere with the sensing mechanism but further enhanced the colour variance by providing high contrast background. ${ }^{\mathbf{1 1 , 8 4}}$ The test result was stable for up to $30 \mathrm{~min}$ and indicates high sensitivity for the epinephrine molecule.

\section{Conclusions}

To the best of our knowledge, the present work is the first established colorimetric detection of epinephrine using an aptamer based approach. The minimum amount of epinephrine detected using AuNPs-apt system was $9 \times 10^{-9} \mathrm{M}$ on a paper based sensor and $0.9 \times 10^{-9} \mathrm{M}$ using UV-visible spectroscopy. While all the parameters tested in this current study played an important role in the functionality of the paper-based sensor, the size and morphology of the AuNPs proved most crucial in controlling the detection limit. It was observed that bigger size particles with greater negative charge are too stable and therefore not suitable for an efficient sensor. This optimized AuNPs-apt sensor approach can be used for the detection of any biomolecule so long as the bound aptamer is designed to detect the target analyte. Successful implementation of this paper-based sensor will provide easy, rapid, portable and inexpensive point-of-care testing which will revolutionize the world of bio-diagnostics and bio-imaging.

\section{Conflicts of interest}

There are no conflicts to declare.

\section{Acknowledgements}

Authors acknowledge the financial support from Florida Department of Agriculture and Consumer Services (FDACS), Contract \#022392. Authors also acknowledge Ankur Gupta, UCF for acquiring HRTEM images.

\section{References}

1 http://www.marketsandmarkets.com/PressReleases/pointof-care-diagnostic.asp, 2016.

2 R. D. Grange, J. P. Thompson and D. G. Lambert, Br. J. Anaesth., 2014, 112, 213-216.

3 M. Vidotti, R. F. Carvalhal, R. K. Mendes, D. C. M. Ferreira and L. T. Kubota, J. Braz. Chem. Soc., 2011, 22, 3-20.

4 W. R. G. Baeyens, S. G. Schulman, A. C. Calokerinos, Y. Zhao, A. M. García Campaña, K. Nakashima and D. De Keukeleire, J. Pharm. Biomed. Anal., 1998, 17, 941-953.

5 Y. C. Chen and W. Y. Lin, Luminescence, 2010, 25, 43-49.

6 S. Zhao, Y. Huang, M. Shi, R. Liu and Y. M. Liu, Anal. Chem., 2010, 82, 2036-2041.

7 M. T. Donato, N. Jimenez, J. V. Castell and M. J. GomezLechon, Drug Metab. Dispos., 2004, 32, 699-706.

8 L. J. Jones, S. T. Yue, C. Y. Cheung and V. L. Singer, Anal. Biochem., 1998, 265, 368-374.

9 G.-H. Chen, W.-Y. Chen, Y.-C. Yen, C.-W. Wang, H.-T. Chang and C.-F. Chen, Anal. Chem., 2014, 86, 6843-6849.

10 D. D. Liana, B. Raguse, J. J. Gooding and E. Chow, Sensors, 2012, 12, 11505-11526.

11 W. Zhao, M. M. Ali, S. D. Aguirre, M. A. Brook and Y. Li, Anal. Chem., 2008, 80, 8431-8437.

12 M. C. Daniel and D. Astruc, Chem. Rev., 2004, 104, 293-346. 13 M. A. El-Sayed, Acc. Chem. Res., 2001, 34, 257-264.

14 N. I. Kovtyukhova and T. E. Mallouk, Chem.-Eur. J., 2002, 8, 4354-4363.

15 D. S. Grubisha, R. J. Lipert, H.-Y. Park, J. Driskell and M. D. Porter, Anal. Chem., 2003, 75, 5936-5943.

16 X. X. Han, Y. Kitahama, T. Itoh, C. X. Wang, B. Zhao and Y. Ozaki, Anal. Chem., 2009, 81, 3350-3355.

17 C. J. Murphy, T. K. Sau, A. M. Gole, C. J. Orendorff, J. Gao, L. Gou, S. E. Hunyadi and T. Li, J. Phys. Chem. B, 2005, 109, 13857-13870.

18 G. Wang, R. J. Lipert, M. Jain, S. Kaur, S. Chakraboty, M. P. Torres, S. K. Batra, R. E. Brand and M. D. Porter, Anal. Chem., 2011, 83, 2554-2561. 
19 R. Elghanian, J. J. Storhoff, R. C. Mucic, R. L. Letsinger and C. A. Mirkin, Science, 1997, 277, 1078-1081.

20 M. Gluodenis and C. A. Foss, J. Phys. Chem. B, 2002, 106, 9484-9489.

21 P. Hanarp, M. Käll and D. S. Sutherland, J. Phys. Chem. B, 2003, 107, 5768-5772.

22 S. Link and M. A. El-Sayed, J. Phys. Chem. B, 1999, 103, 84108426.

23 S. Schultz, D. R. Smith, J. J. Mock and D. A. Schultz, Proc. Natl. Acad. Sci. U. S. A., 2000, 97, 996-1001.

24 I. O. Sosa, C. Noguez and R. G. Barrera, J. Phys. Chem. B, 2003, 107, 6269-6275.

25 K. Ai, Y. Liu and L. Lu, J. Am. Chem. Soc., 2009, 131, 94969497.

26 D. Aili, R. Selegard, L. Baltzer, K. Enander and B. Liedberg, Small, 2009, 5, 2445-2452.

27 S. Krishnan, V. Mani, D. Wasalathanthri, C. V. Kumar and J. F. Rusling, Angew. Chem., Int. Ed. Engl., 2011, 50, 11751178.

28 X. Xie, W. Xu and X. Liu, Acc. Chem. Res., 2012, 45, 15111520.

29 A. W. Clark and J. M. Cooper, Angew. Chem., 2012, 124, 36223626.

30 C. A. Mirkin, R. L. Letsinger, R. C. Mucic and J. J. Storhoff, Nature, 1996, 382, 607.

31 T. Mori and M. Maeda, Polym. J., 2002, 34, 624-628.

32 K. Sato, K. Hosokawa and M. Maeda, J. Am. Chem. Soc., 2003, 125, 8102-8103.

33 J. Liu and Y. Lu, J. Am. Chem. Soc., 2004, 126, 12298-12305. 34 X. Xue, F. Wang and X. Liu, J. Am. Chem. Soc., 2008, 130, 3244-3245.

35 S. Kim, J. W. Park, D. Kim, D. Kim, I. H. Lee and S. Jon, Angew. Chem., 2009, 121, 4202-4205.

36 Z. Mei, H. Chu, W. Chen, F. Xue, J. Liu, H. Xu, R. Zhang and L. Zheng, Biosens. Bioelectron., 2013, 39, 26-30.

37 N. Zhou, J. Wang, J. Zhang, C. Li, Y. Tian and J. Wang, Talanta, 2013, 108, 109-116.

38 Y. S. Kim, J. H. Kim, I. A. Kim, S. J. Lee, J. Jurng and M. B. Gu, Biosens. Bioelectron., 2010, 26, 1644-1649.

39 K.-M. Song, E. Jeong, W. Jeon, M. Cho and C. Ban, Anal. Bioanal. Chem., 2012, 402, 2153-2161.

40 A. D. Ellington and J. W. Szostak, Nature, 1992, 355, 850.

41 J. Abelson, Science, 1990, 249, 488.

42 H. Sun and Y. Zu, Molecules, 2015, 20, 11959-11980.

43 S. K. Ghosh and T. Pal, Chem. Rev., 2007, 107, 4797-4862.

44 C. J. Murphy, A. M. Gole, J. W. Stone, P. N. Sisco, A. M. Alkilany, E. C. Goldsmith and S. C. Baxter, Acc. Chem. Res., 2008, 41, 1721-1730.

45 F. E. R. Simons, J. Allergy Clin. Immunol., 2004, 113, 837-844. 46 T. Härle, K. Kronberg, H. Nef, H. Möllmann and A. Elsässer, Clin. Res. Cardiol., 2011, 100, 471-473.

47 A. Sheikh, Y. A. Shehata, S. G. A. Brown and F. E. R. Simons, Cochrane Database of Systematic Reviews, 2008, 4, 1-9.

48 E. D. Bergmann and Z. Goldschmidt, J. Med. Chem., 1968, 11, 1121-1125.

49 S. Alpat, K. Ozdemir and S. Alpat, J. Sens., 2016, 2016, 1-99.
50 X. Lu, Y. Li, J. Du, X. Zhou, Z. Xue, X. Liu and Z. Wang, Electrochim. Acta, 2011, 56, 7261-7266.

51 F. C. Moraes, D. L. Golinelli, L. H. Mascaro and S. A. Machado, Sens. Actuators, B, 2010, 148, 492-497.

52 Z. Yang, G. Hu, X. Chen, J. Zhao and G. Zhao, Colloids Surf., $B, 2007,54,230-235$.

53 V. Carrera, E. Sabater, E. Vilanova and M. A. Sogorb, J. Chromatogr. B: Anal. Technol. Biomed. Life Sci., 2007, 847, 88-94.

54 M. A. Fotopoulou and P. C. Ioannou, Anal. Chim. Acta, 2002, 462, 179-185.

55 M. Sorouraddin, J. Manzoori, E. Kargarzadeh and A. H. Shabani, J. Pharm. Biomed. Anal., 1998, 18, 877-881.

56 S. Wei, G. Song and J.-M. Lin, J. Chromatogr. A, 2005, 1098, 166-171.

57 J. Du, L. Shen and J. Lu, Anal. Chim. Acta, 2003, 489, 183-189. 58 X. Zheng, Z. Guo and Z. Zhang, Anal. Chim. Acta, 2001, 441, 81-86.

59 W. K. Adeniyi and A. R. Wright, Spectrochim. Acta, Part A, 2009, 74, 1001-1004.

60 J. Yang, G. Zhang, X. Wu, F. Huang, C. Lin, X. Cao, L. Sun and Y. Ding, Anal. Chim. Acta, 1998, 363, 105-110.

61 Y.-F. Huang, H.-T. Chang and W. Tan, Anal. Chem., 2008, 80, 567-572.

62 H. R. Sim, A. W. Wark and H. J. Lee, Analyst, 2010, 135, 25282532.

63 S. J. Zhen, C. Z. Huang, J. Wang and Y. F. Li, J. Phys. Chem. C, 2009, 113, 21543-21547.

64 S. Niu, Z. Lv, J. Liu, W. Bai, S. Yang and A. Chen, PLoS One, 2014, 9, e109263.

65 M. M. Maye, L. Han, N. N. Kariuki, N. K. Ly, W.-B. Chan, J. Luo and C.-J. Zhong, Anal. Chim. Acta, 2003, 496, 17-27.

66 V. Behaj, J. I. Cohen and R. Engel, Anal. Lett., 2002, 35, 17151720.

67 D. A. Bruzewicz, M. Reches and G. M. Whitesides, Anal. Chem., 2008, 80, 3387-3392.

68 E. Metcalf, M. Morgan and P. Dean, J. Chromatogr. A, 1982, 235, 501-506.

69 A. J. Nozik and R. Memming, J. Phys. Chem., 1996, 100, 13061-13078.

70 H. H. Patel and A. R. Kharat, Int. J. Pharm. Sci. Res., 2012, 3, 5015-5023.

71 J. Wang, A. Munir, Z. Li and H. S. Zhou, Biosens. Bioelectron., 2009, 25, 124-129.

72 Y. Zheng, Y. Wang and X. Yang, Sens. Actuators, B, 2011, 156, 95-99.

73 Y. Xue, X. Li, H. Li and W. Zhang, Nat. Commun., 2014, 5, 4348.

74 H. Häkkinen, Nat. Chem., 2012, 4, 443-455.

75 V. S. R. Aaryasomayajula, T. Severs, K. Ghosh, R. DeLong, X. Zhang, S. Talapatra and A. K. Wanekaya, J. Nanomed. Nanotechnol., 2014, 5, 1.

76 H. Li and L. Rothberg, Proc. Natl. Acad. Sci. U. S. A., 2004, 101, 14036-14039.

77 C. Yang, Y. Wang, J.-L. Marty and X. Yang, Biosens. Bioelectron., 2011, 26, 2724-2727. 
78 S. Basu, S. K. Ghosh, S. Kundu, S. Panigrahi, S. Praharaj, S. Pande, S. Jana and T. Pal, J. Colloid Interface Sci., 2007, 313, 724-734.

79 A. N. Afrooz, S. T. Sivalapalan, C. J. Murphy, S. M. Hussain, J. J. Schlager and N. B. Saleh, Chemosphere, 2013, 91, 93-98. 80 A. V. Kyrylyuk and A. P. Philipse, Phys. Status Solidi A, 2011, 208, 2299-2302.

81 J. F. Rudge, M. B. Holness and G. C. Smith, Contrib. Mineral. Petrol., 2008, 156, 413-429.
82 G.-H. Chen, W.-Y. Chen, Y.-C. Yen, C.-W. Wang, H.-T. Chang and C.-F. Chen, Anal. Chem., 2014, 86, 6843-6849.

83 I. S. Wittstein, D. R. Thiemann, J. A. Lima, K. L. Baughman, S. P. Schulman, G. Gerstenblith, K. C. Wu, J. J. Rade, T. J. Bivalacqua and H. C. Champion, N. Engl. J. Med., 2005, 352, 539-548.

84 J. Liu, D. Mazumdar and Y. Lu, Angew. Chem., 2006, 118, 8123-8127. 